\title{
ON GENERALIZED CHERN CLASSES AND CHERN NUMBERS \\ OF IRREDUCIBLE COMPLEX ALGEBRAIC VARIETIES WITH ARBITRARY SINGULARITIES
}

\author{
WEN-TSUN WU \\ MMKL, Academy of Mathematics and System Sciences, Academia Sinica \\ Beijing, 100080, P.R. China
}

For an irreducible complex algebraic variety which is non-singular there is a naturally associated tangent bundle with tangent spaces as fibers. For such bundles the late Professor Chern Shingshen introduced the so-called Chern classes and Chern numbers which revolutionized the modern mathematics. Moreover, Chern pointed out that for algebraic varieties these Chern classes enjoy an algebrico-geometrical character, cf. his classical papers $[\mathrm{CH} 1,2]$.

Now for an irreducible complex algebraic variety with singularities no such tangent bundle is defined. To meet the difficulties mathematicians had applied some blow-up process to get some non-singular varieties and Chern classes and Chern numbers are then introduced in this ingenious way, cf. notably [MacPh]. In any way it seems that there are few ways of determining such generalized Chern classes and Chern numbers in concrete cases. In the present article dedicated to the late Professor Ch. Ehresmann we shall give a simple method of introducing generalized Chern classes and Chern numbers for irreducible complex algebraic varieties with arbitray singularities which are computable in some natural sense and may be easily determined in concrete cases.

To see this let us consider an irreducible complex algebraic variety $V_{d}$ of dimension $d$ in a complex projective space $P_{\mathbf{C}}^{n}$ of dimension $n$. Let us take an arbitrary generic point $G$ of $V_{d}$. Then $G$ is a regular point of $V_{d}$ and has the well-defined tangent space $T_{G}$ of dimension $d$ through that point. The pair $\left(G, T_{G}\right)$ may be considered as a point in the composite Grassmannian $G R(n ; 0, d)$ of composite elements consisting of pairs of an arbitrary point as well as an arbitrary $d$-dimensional linear space through that point in the complex projective space $P_{\mathbf{C}}^{n}$. The pair $\left(G, T_{G}\right)$ will then determine a subvariety of

This paper is dedicated to the memory of Professor Ch. Ehresmann, who was my advisor for the Docteur ès Sciences thesis in 1949.

The paper is in final form and no version of it will be published elsewhere. 
dimension $d$ in the composite Grassmannian $G R(n ; 0, d)$ with $\left(G, T_{G}\right)$ as a generic point which will be denoted as $\tilde{V}$.

According to Ehresmann (see $[\mathrm{EH}]$ ), the total homology group with complex coefficients of $G R(n ; 0, d)$ may be described as follows. In the complex projective space $P_{\mathbf{C}}^{n}$ let us consider an arbitrary sequence $\mathbf{S}$ of linear subspaces $L_{i}$ of dimension $i=0,1, \ldots, n$ such that

$$
\mathbf{S}: L_{0} \subset L_{1} \subset \ldots \subset L_{n-1} \subset L_{n}=P_{\mathbf{C}}^{n} .
$$

Consider a sequence of $d$ integers $b_{i}, i=0,1, \ldots, d$ such that

$$
0 \leq b_{0}<b_{1}<\ldots<b_{d-1}<b_{d} \leq n .
$$

Then the subvariety of $G R(n ; 0, d)$ consisting of composite elements $\left(L_{0}^{\prime}, L_{d}^{\prime}\right)$ with $L_{0}^{\prime}$ a point in some $L_{i}, i \in\{0,1, \ldots, n\}$, and $L_{d}^{\prime}$ a $d$-dimensional linear subspace through $L_{0}^{\prime}$ for which

$$
\operatorname{Dim}_{\mathbf{C}}\left(L_{d}^{\prime} \cap L_{b_{j}}\right) \geq j, \quad j=0,1, \ldots, d,
$$

will be called a Schubert cycle of Ehresmann symbol

$$
E=\left[\begin{array}{lllll}
b_{i} \mid b_{0} & b_{1} & \ldots & b_{d}
\end{array}\right] .
$$

According to Ehresmann such cycles will belong to the same homology class $C_{E}$ of $G R(n ; 0, d)$ irrespective of the chosen linear subspaces $L_{i}^{\prime}$ and the class is of dimension

$$
\operatorname{Dim}_{\mathbf{C}} C_{E}=\sum_{j}\left(b_{j}-j\right)+b_{i} .
$$

We shall call accordingly such a homology class $C_{E}$ of the composite Grassmannian an Ehresmann class of Ehresmann symbol E. In particular, the composite Grassmannian itself is a cycle of Ehresmann symbol

$$
G R(n ; 0, d)=[n \mid(n-d, n)],
$$

in which $(i, j)$ for $i \leq j$ will stand for the sequence of successive integers $i, i+1, \ldots, j$. The dimension of $G R(n ; 0, d)$ is thus, by (5),

$$
\operatorname{Dim}_{\mathbf{C}} G R(n ; 0, d)=(n-d) *(d+1)+d=D \text {, say. }
$$

Now the composite Grassmannian $G R(n ; 0, d)$ is naturally a compact complex differential manifold so that both duality and intersections of homology classes are well-defined. Let us define for the cycle $E$ in (4) its dual

$$
\delta E=\left[n-b_{i} \mid n-b_{d} \ldots n-b_{1} n-b_{0}\right],
$$

then the induced duality operator $\delta_{*}$ of homology classes will be given by

$$
\delta_{*} C_{E}=C_{\delta E} .
$$

The intersections in the composite Grassmannian are rather complicated and we shall restrict ourselves to the case of codimensions $\leq d$ or dimensions $\geq D-d$. In these dimensions there are two kinds of cycles $\delta P, \delta Q_{h}, 0 \leq h \leq d$, of particular importance for which

$$
P=[1 \mid(0, d)], \quad Q_{h}=[0 \mid(0, d-1), d+h]
$$


In fact, the intersection ring of $G R(n ; 0, d)$ in the above dimensions is generated by the above cycles and we have for example ( means homologous cycles)

$$
\begin{aligned}
& \delta\left[i \mid(0, i), b_{i+1}, \ldots, b_{d}\right] \sim \delta\left[0 \mid(0, i), b_{i+1}, \ldots, b_{d}\right] *(\delta P)^{i}, \\
& \delta\left[0 \mid a_{0}, \ldots, a_{d}\right] * \delta\left[0 \mid b_{0}, \ldots, b_{d}\right] \sim \sum_{c} \delta\left[0 \mid c_{0}, \ldots, c_{d}\right],
\end{aligned}
$$

in which the summation is over $c=\left(c_{0}, \ldots, c_{d}\right)$ such that in the ordinary Grassmann variety $G R(n ; d)$ we shall have the intersection formula

$$
\delta\left[a_{0}, \ldots, a_{d}\right] * \delta\left[b_{0}, \ldots, b_{d}\right] \sim \sum_{c} \delta\left[c_{0}, \ldots, c_{d}\right] .
$$

See for more details [H-P], v.2, Chap. 14.

Now for any pair $\left(P, L_{P}\right)$ consisting of a point $P$ and a $d$-dimensional linear space $L_{P}$ through $P$ in the complex projective space $P_{\mathbf{C}}^{n}$ the restriction of the map $\iota_{*}:\left(P, L_{P}\right) \rightarrow$ $P$ to $\tilde{V}$ will induce a homomorphism of homology groups $\iota_{*}: H_{*}(\tilde{V}, \mathbf{C}) \rightarrow H_{*}(V, \mathbf{C})$. Consider now in $G R(n ; 0, d)$ an arbitrary cycle $C_{E}$ of codimension $\geq D-d$ and $\leq D$ of Ehresmann symbol $E$ which may be taken to be in general position with $\tilde{V}$. The intersection of $C_{E}$ with $\tilde{V}$ will give then a cycle of dimension $\geq 0$ and $\leq d$. The image under $\iota_{*}$ of the homology class of such cycles is then well-defined and will be called an Ehresmann-Chern class or a generalized Chern class of Ehresmann symbol E. In the case of dimension 0 these will give the respective Ehresmann-Chern numbers or generalized Chern numbers. Owing to works of J. A. Todd and P. B. Gamkrelidze there are linear combinations of the above Ehresmann-Chern or generalized Chern classes and numbers which will give the ordinary Chern classes and numbers when the variety in question has no singularities. As the explicit expressions of Todd-Gamkrelidze are rather complicated we shall not give them here.

Now in the case of algebraic varieties without singularities it is well-known that Chern numbers satisfy some inequalities. For example, for certain hypersurfaces in a 3-dimensional complex projective space there is the celebrated Miyaoka-Yau inequality (see [MIY] and [YAU]), viz.

$$
\left(c_{1}\right)^{2} \leq 3 c_{2}
$$

Applying our method it may however be easily proved by simple computations that this inequality holds for any hypersurface with arbitrary singularities in 3-dimensional complex projective space without any restriction. Other kinds of inequalities also exist between such (generalized) Chern numbers in higher dimensional case. In fact, Shi He discovered and proved a lot of equalities and inequalities among such generalized Chern numbers for which we refer to his papers [SHI1,2].

Finally, let us point out that late Professor Chow Weiliang had introduced for arbitrary complex algebraic varieties various notions of algebraic equivalence classes and groups. What we said above may be naturally interpreted as such equivalence classes and groups in purely algebrico-geometrical sense without intervention of any topological concepts. For these we refer to related writings in the references. 


\section{References}

[CH1] S. S. Chern, Characteristic classes of Hermitian manifolds, Annals of Math. 47 (1946), 88-121.

[CH2] S. S. Chern, On the characteristic classes of complex sphere bundles and algebraic varieties, Amer. J. Math. 75 (1953), 565-577.

[CHOW1] W. L. Chow, Algebraic varieties with rational dissections, Proc. Nat. Acad. Sci. U.S.A. 42 (1956), 116-119.

[CHOW2] W. L. Chow, On the equivalence classes of cycles in an algebraic variety, Annals of Math. 64 (1956), 450-479.

[EH] Ch. Ehresmann, Sur la topologie de certaines espaces homogènes, Annals of Math. 35 (1934), 398-443.

[Gam] P. B. Gamkrelidze, Chern cycles of complex algebraic manifolds, Izv. Acad. Scis. SSSR Math. Series 20 (1956), 685-706 (in Russian).

[H-P] W. V. D. Hodge and D. Pedoe, Methods of Algebraic Geometry, Vol. 2, Camb. Univ. Press, 1952.

[MacPh] R. D. MacPherson, Chern classes for singular algebraic varieties, Annals of Math. 100 (1974), 423-432.

[MIY] Y. Miyaoka, On the Chern numbers of surfaces of general type, Invent. Math. 42 (1977), 225-237.

[SHI1] H. Shi, On Chern characters of algebraic hypersurfaces with arbitrary singularities, Acta Math. Sinica New Series 4 (1988), 289-360.

[SHI2] H. Shi, Chern classes of algebraic varieties with singularity, in: Singularity Theory, World Scientific, Singapore, 1995, 705-730.

[Todd] J. A. Todd, The geometrical invariants of algebraic loci, Proc. London Math. Soc. 43 (1937), 127-138.

[WU1] W. T. Wu, On Chern characteristic systems of an algebraic variety, Shuxue Juzhan 8 (1965), 395-401 (in Chinese).

[WU2] W. T. Wu, On Chern numbers of algebraic varieties with arbitrary singularities, Acta Math. Sinica New Series 3 (1987), 227-236.

[WU3] W. T. Wu, Mathematics Mechanization: Mechanical Geometry Theorem-Proving, Mechanical Geometry Problem-Solving and Polynomial Equations-Solving, Science Press and Kluwer Academic Publishers, 2000, Chap. 5, $\S \S 1-3$.

[YAU] S. T. Yau, Calabi's conjecture and some new results in algebraic geometry, Proc. Nat. Acad. Sci. U.S.A. 74 (1977), 1798-1799. 


\section{MINICOURSE}


